\title{
THE ANGULAR STRUCTURES OF SOME COMPACT SOURCES
}

\author{
H. P. PALMER \\ Nuffield Radio Astronomy Laboratories, Jodrell Bank, Cheshire, Great Britain
}

From 1965 to 1967 radio link interferometers were operated between Jodrell Bank and Malvern, at wavelengths of 21,11 , and $6 \mathrm{~cm}$. Preliminary results were published by Adgie et al. (1965), Barber et al. (1966), Palmer et al. (1967) and Miley et al. (1967). The full analysis of these data has now been completed. Donaldson et al. (1969) report that six sources were unresolved, and they set size limits in the range 0.1 to 0.01 arc sec. Seven sources were found to contain unresolved components, and similar but less

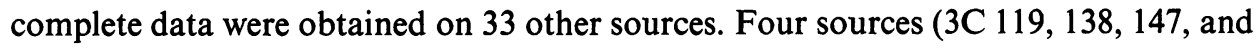
237) were shown to have double or more complex structures. (Donaldson and Smith, 1970; Donaldson et al., 1970). The cores of 3C 274 and four other sources were shown to have elongations in the range 0.3 to $0.03 \mathrm{arcsec}$. One of these is $3 \mathrm{C} 287$, and when the data on this source are combined with those of Clark et al. (1968) at $1660 \mathrm{MHz}$ it appears that this source is a double of separation 0.08 arc sec in position angle $025^{\circ}$. This differs from the interpretation given by Clark et al. Some new data were also obtained on the well known double sources 3C 405 and 1938-15.

Critchley and Palmer (1971) will report shortly an investigation of the angular structures at $408 \mathrm{MHz}$ of 105 radio sources catalogued at Parkes in the declination range $-13^{\circ}$ to $-17^{\circ}$. Most of these sources have fluxes in the range 1-6 flux units. The angular structures found have been compared with the information available for a group of more intense sources from the 3CR catalogue. No general trend towards smaller angular sizes can be seen amongst the less intense sources. A comparison of the axial ratios of the double sources in the two groups suggests, however, that widely separated double sources having compact components are two or three times more abundant amongst the group of less intense sources. As one studies sources of decreasing flux density, confusion will eventually produce this effect, but in this data the compact components are 30-100 arc sec apart and they radiate more than $0.5 \mathrm{f}$.u. Chance coincidences could produce only about one in ten of the wide-spaced double sources found in this sample.

Several explanations have been suggested for this apparent increase in the proportion of sources with high axial ratios. It may arise for one or both of two observational reasons. The angular structures of the two groups were measured with instruments of very different resolving power, and the Parkes sources were selected at $408 \mathrm{MHz}$, while the $3 \mathrm{CR}$ sample was catalogued at $178 \mathrm{MHz}$. If the effect is confirmed for two groups of sources selected in the same way, and observed with the same instrument, it suggests that, if these faint double sources have red-shifts greater than 1 , and hence separations greater than $1.8 \mathrm{Mpc}$, these small components must have remained confined for longer than $6 \times 10^{6} \mathrm{yr}$. However, these sources have the same angular scale as the more intense sources, so their red-shifts may be nearer 0.3 . 


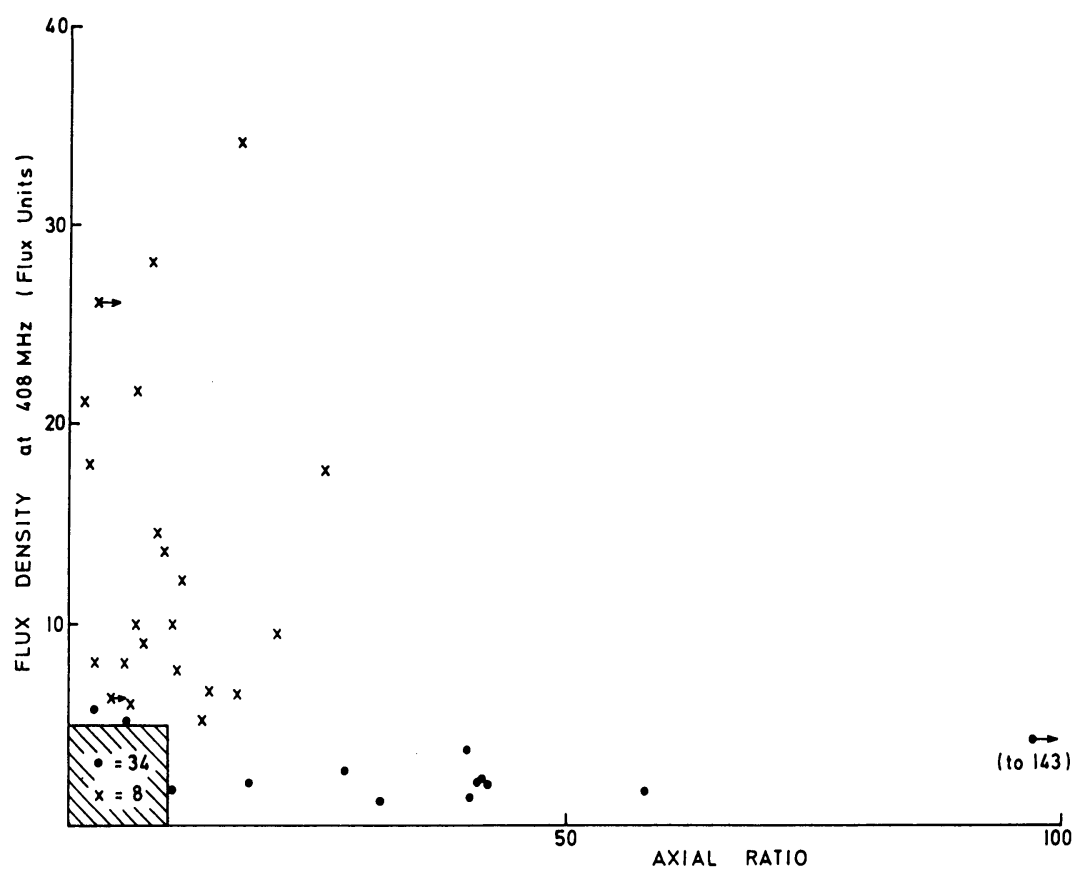

Fig. 1. The axial ratio (separation of components/component size) plotted against flux at $408 \mathrm{MHz}$, for the 77 double sources found in groups which are complete to 3 , and to 1 flux unit respectively. (42 sources fall in the shaded area near the origin.) - The double sources found in a complete sample of 105 Parkes sources $\left(\delta-13^{\circ}\right.$ to $\left.-17^{\circ}\right) ; \times$ The doubles found in a complete sample of $1203 \mathrm{C}$ sources $\left(\delta>35^{\circ} b^{\prime \prime}>10^{\circ}\right)$.

The components of classical double radio sources may contain 'cores' which continue to emit after the extended regions have decayed.

\section{References}

Adgie, R. L., Gent, H., Slee, O. B., Frost, A. D., Palmer, H. P., and Rowson, B.: 1965, Nature 208, 275.

Barber, D., Donaldson, W., Miley, G. K., and Smith, H.: 1966, Nature 209, 753.

Clark, B. G., Kellermann, K. I., Bare, C. C., Cohen, M. H., and Jauncey, D. L.: 1968, Astrophys. J. 153, 705.

Critchley, J. and Palmer, H. P.: 1971, Monthly Notices Roy. Astron. Soc., in preparation.

Donaldson, W., Miley, G. K., Palmer, H. P., and Smith, H.: 1969, Monthly Notices. Roy. Astron. Soc. 146, 213.

Donaldson, W., and Smith, H.: 1970, Monthly Notices Roy. Astron. Soc., in preparation.

Donaldson, W. and Miley, G. K.: 1970, Monthly Notices Roy. Astron. Soc., in preparation.

Miley, G. K., Rickett, B. J., and Gent, H.: 1967, Nature 216, 974.

Palmer, H. P., Rowson, B., Anderson, B., Donaldson, W., Miley, G. K., Gent, H., Adgie, R. L. Slee, O. B., and Crowther, J. H.: 1967, Nature 213, 789. 